\title{
New advantageous tool in single incision laparoscopic cholecystectomy: the needle grasper
}

\author{
Turgut Donmez ${ }^{1}$, Sinan Uzman², Sina Ferahman ${ }^{3}$, Suleyman Demiryas ${ }^{3}$, Engin Hatipoglu ${ }^{3}$, Server Sezgin Uludag ${ }^{3}$, \\ Dogan Yildirim ${ }^{4}$ \\ ${ }^{1}$ Department of General Surgery, Lutfiye Nuri Burat State Hospital, Istanbul, Turkey \\ ${ }^{2}$ Department of Anesthesiology and Reanimation, Haseki Training and Research Hospital, Istanbul, Turkey \\ ${ }^{3}$ Department of General Surgery, Medical Faculty, Istanbul University Cerrahpasa, Istanbul, Turkey \\ ${ }^{4}$ Department of General Surgery, Haseki Training and Research Hospital, Istanbul, Turkey
}

Videosurgery Miniinv 2016; 11 (1): 38-43

DOI: $10.5114 /$ wiitm.2016.58978

\begin{abstract}
Introduction: During single-incision laparoscopic cholecystectomy (SILC), the gallbladder is suspended with stitches, resulting in perforation risk and difficulty in exploration.

Aim: We used the needle grasper in SILC to hang and manipulate the gallbladder.

Material and methods: Sixty-five patients (43 female, 22 male) who underwent SILC between December 2013 and December 2014 were analyzed retrospectively for patient demographics, duration of operation, laparotomy or conventional laparoscopy necessity, drain use, complications, and hospital stay periods. To place the SILC port (Covidien, Inc.), the needle grasper was inserted at the right upper abdominal quadrant without an incision to hang and manipulate the gall-bladder.

Results: The mean age was $47.9 \pm 13.068$ years; the mean body mass index (BMI) was $26.94 \pm 3.913 \mathrm{~kg} / \mathrm{m}^{2}$. ASA scores were 1, 2, and 3. Two patients with high BMI with additional trocar use were excluded. The operations were completed without any additional trocar in 59 patients. The mean operation time was $89 \pm 22.41 \mathrm{~min}$. Eighteen patients required a drain; all were discharged after drain removal. One patient needed re-hospitalization and percutaneous drainage and was discharged on the $9^{\text {th }}$ day. Fifty-three patients were discharged on the $1^{\text {st }}$ post-operative day. Eleven patients with drains were discharged on the $2^{\text {nd }}$ day, and 1 was discharged on the $7^{\text {th }}$ day. The mean hospital stay period was $1.26 \pm 0.815$ days.

Conclusions: The main difficulty of SILC is to manipulate hand tools because the triangulation principle of laparoscopy use is not possible in SILC. Inserting a needle grasper into the abdominal cavity at the right subcostal area to manipulate the gallbladder helps and does not leave a visible scar.
\end{abstract}

Key words: laparoscopic cholecystectomy, single port, needle grasper.

\section{Introduction}

Minimal invasive techniques that modify laparoscopic operations are widely used. Surgeons all over the world have accepted minimal invasive techniques since the end of the 1980s [1]. Single-incision laparoscopic surgery (SILS), natural orifice transluminal endoscopic surgery (NOTES) or trans-umbilical single-port laparoscopic surgery (TUSPL) techniques are used widely in almost every surgical clinic today.

The number of single-incision laparoscopic cholecystectomies performed has significantly increased

\section{Address for correspondence}

Turgut Donmez PhD, Department of General Surgery, Lutfiye Nuri Burat State Hospital, 50. yıl Mh. A, blok 2106 sok. Sultangazi,

34300 Istanbul, Turkey, phone: +90 5347400967, e-mail: surgeont73@hotmail.com 
since its introduction [2]. The desire for the least scarring, the least pain, and the shortest hospital stay have been given as reasons for this increase.

Is it feasible to make an operational technique more difficult for the sake of patient satisfaction? Actually, cosmetic considerations have been becoming more important in this era as well as treatment. Patients want less scar tissue and shorter hospital stays. Thus, the future will be characterized by surgeons who perform proper minimally invasive operations with minimal scarring.

Cholecystectomy is one of the most frequently performed operations in surgical clinics today [3]. Since Navarra performed the first single-port laparoscopic cholecystectomy in 1997, different techniques have been used to perform this operation [4]; however, a consensus has not been reached as to which is the best [5]. One of the most important reasons for the use of various methods is due to the use of different high-technology tools by different groups for different aims. During the first single port cholecystectomy operation, the gallbladder was hung to the abdominal wall with traction stitches in 1997 [4]. Even though it is one of the most widely used procedures, it has some disadvantages, such as risk of gallbladder perforation and difficulty in exploration.

\section{Aim}

This study presents our experience with the needle grasper in SILS cholecystectomy. We used the needle grasper to close the trocar hole on fascia in laparoscopic operations. A needle grasper is shown in Photo 1 along with the hung gallbladder. The needle grasper also allowed us to manipulate the gallbladder.

\section{Material and methods}

We analyzed the patients admitted to the general surgery outpatient or emergency department with abdominal pain or dyspeptic complaints between December 2013 and December 2014, retrospectively. This study was carried out in a 100-bed State Hospital. This is a retrospective clinical study that was performed by scanning the files of operations and patients. All operations were performed by experienced surgeons after obtaining detailed informed consent. Sixty-one patients (41 female, 20 male) who underwent single port cholecystectomy because of cholelithiasis, gallbladder polyp, or gallbladder sludge were first included in the study. Informed consent was obtained from each patient included in the study, and the study protocol confirms to the ethical guidelines of the Declaration of Helsinki as reflected in a priori approval by the institution's human research committee. The study was approved by the Local Hospital Ethics Committee (date: April 22, 2015 no. 214).

All patients underwent abdominal ultrasound, and benign gallbladder disease was confirmed. Patients with abdominal surgery, pregnant women, those with bleeding diathesis, gallbladder cancer, and patients with a diagnosis of acute cholecystitis study were excluded. All operations were performed electively.

All patients were analyzed in terms of patient demographics, duration of operation, laparotomy or conventional laparoscopy necessity, drain usage, complications, and hospital stay periods.

All patients were operated on under general anesthesia by the same surgeon using the same technique.

Patients were laid on the operation table in the prone position with arms open and legs closed. The screen and the $1^{\text {st }}$ assistant were at the right upper side of the patient and the surgeon on the left. A video scope assistant and nurse were at the right lower side (Figure 1). First-generation cephalosporin was used intravenously for prophylaxis just before the surgery.

A conventional $2 \mathrm{~cm}$ vertical SILC incision was performed on the skin at the umbilicus until the linea alba. The intra-abdominal cavity was reached between the rectus abdominis muscles, and a flexible SILC port (Covidien, Inc.) was placed. A pneumoperitoneum of $12 \mathrm{~mm} \mathrm{Hg}$ was formed with $\mathrm{CO}_{2}$. The patient was positioned in the reverse Trendelenburg
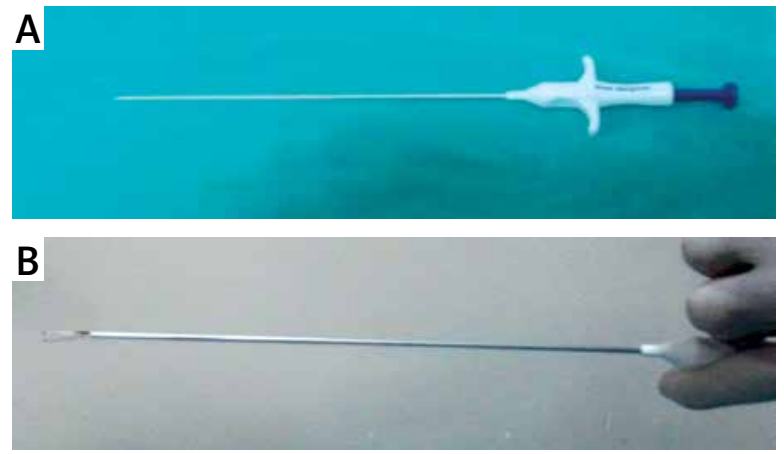

Photo 1. A - Blue needle grasper. B - After pushing the button of the blue needle grasper, the holder part comes out 


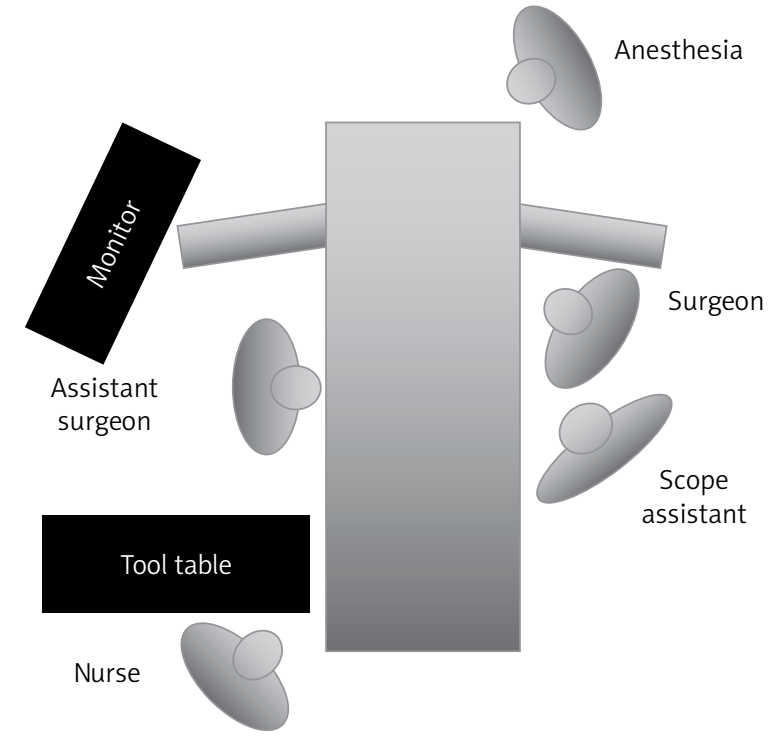

Figure 1. Operation room and table order for single port laparoscopic cholecystectomy

position. $\mathrm{A} 10 \mathrm{~mm} 0^{\circ}$ endoscope and two $5 \mathrm{~mm}$ working hand-tools were inserted through the SILS port. Two patients with body mass index (BMI) $38 \mathrm{~kg} / \mathrm{m}^{2}$ and $43 \mathrm{~kg} / \mathrm{m}^{2}$ necessitated additional trocar use because of the insufficient hand tools. These 2 cases were excluded from the study. The needle grasper (Blue Surgical, Inc.) was inserted at the right upper abdominal quadrant in the remaining 59 patients without making an incision (Photo 2). The gallbladder fundus was grasped with a needle grasper and hung in 59 patients without difficulty. The second assistant positioned the gallbladder using the nee-
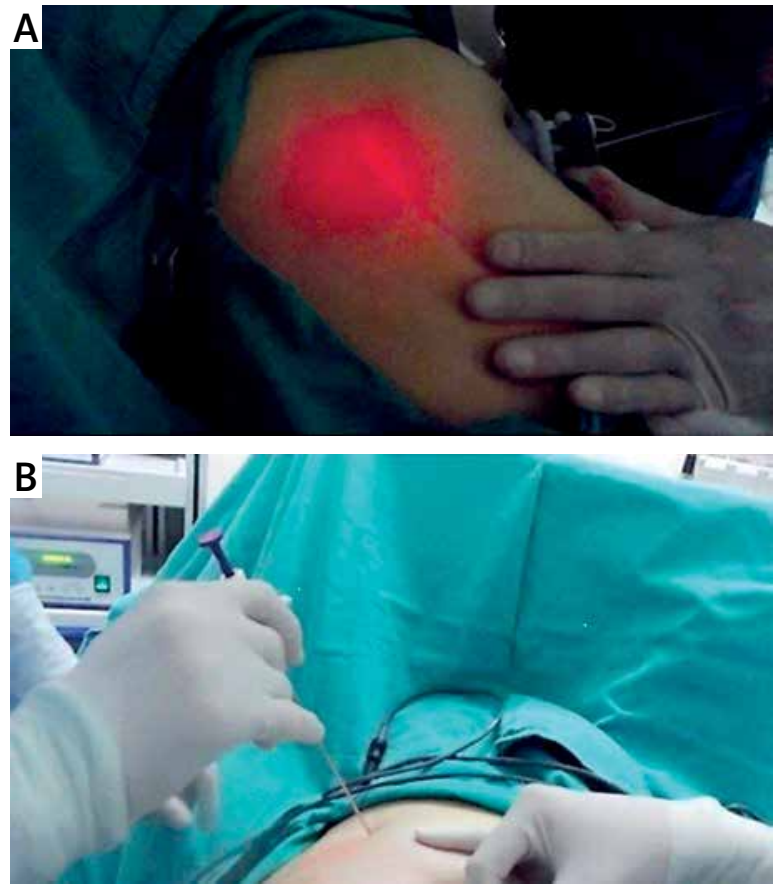

Photo 2. A - Guiding the blue needle grasper device for appropriate entrance point by optical light. B - Entrance of the blue needle grasper device

dle grasper. The surgeon grasped the gallbladder at its neck with a grasper, and its serosa was incised by using a hook; the Calot triangle was also clarified. Especially during this dissection, the needle grasper was effectively used to turn the gallbladder either to medial or lateral directions (Photo 3). The cystic duct and artery were dissected, separately clipped

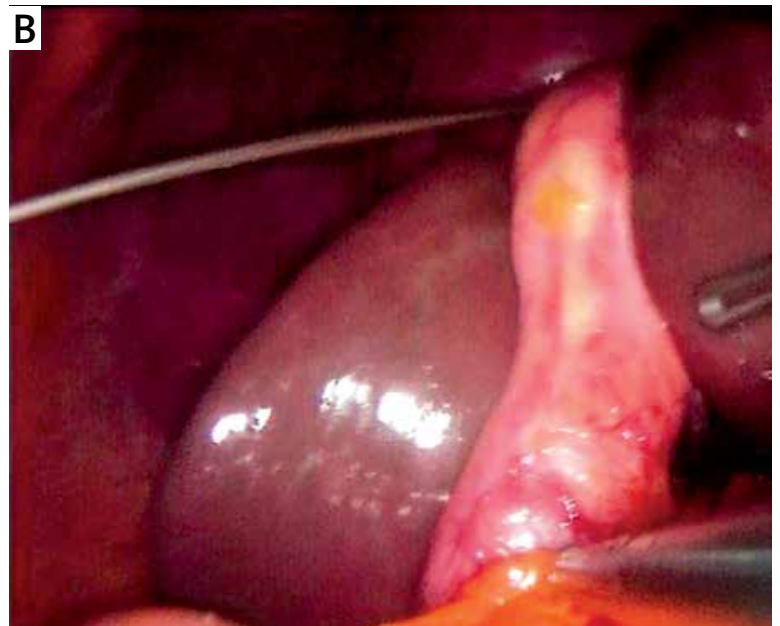

Photo 3. A - Holding the fundus of the gallbladder by the blue needle grasper device. B - Retraction of the gallbladder upward by the needle grasper 
with a $5 \mathrm{~mm}$ endoscopic clip, and cut. The gallbladder was separated from the liver bed, and the endoscopic needle was taken out. The needle grasper entry port was dressed and did not require any sutures. The gallbladder was grasped with a clinch and taken out of the abdomen along with the SILS Port. Fascia defects and skin were closed with sutures. No additional trocar was needed in these 59 patients. All the patients were given NSAIDs for pain after the operation. All patients were asked to come at the $7^{\text {th }}$ and $15^{\text {th }}$ post-operative day and one month later for follow-up.

\section{Results}

Fifty-nine (40 female, 19 male) patients were included in the study. The mean age was $47.9 \pm 13.068$ years (min: 21; max: 75), and the mean BMI was $26.94 \pm 3.913 \mathrm{~kg} / \mathrm{m}^{2}$ (min: 19.6; max: 43.25). Thirtyfive patients were ASA 1, 21 were ASA 2, and 3 patients were ASA 3.

Two patients with high BMI $\left(38.06 \mathrm{~kg} / \mathrm{m}^{2}\right.$ and $43.25 \mathrm{~kg} / \mathrm{m}^{2}$, respectively) required additional trocar use due to technical insufficiency and were therefore excluded from the study. The operations were completed without any additional trocar or complication in the remaining 59 patients. The mean operation time was $89 \pm 22.41 \mathrm{~min}$ ( $\mathrm{min}: 50 \mathrm{~min}$, max: $185 \mathrm{~min}$ ).

Eleven patients required hemovac drain placement into the gallbladder bed due to minor hemorrhage leakage from the liver bed. Bile leakage was observed in only 1 (1.69\%) patient. All the patients were discharged after removal of the drain. One patient experienced abdominal pain after discharge, and ultrasonography (USG) revealed fluid collection at the pouch of Douglas. The patient was not diagnosed for the cause of the leakage. The patient was hospitalized, and a percutaneous drainage catheter was placed by interventional radiologists into the lodge; $300 \mathrm{ml}$ of bile-stained fluid was drained. The biliary discharge stopped spontaneously during observation, and the patient was discharged on the $9^{\text {th }}$ day. Forty-eight patients were discharged on the $1^{\text {st }}$ post-operative day. Eleven patients with drains were discharged on the $2^{\text {nd }}$ postoperative day. The mean hospitalization period was $1.26 \pm 0.815$ days (min: 1 day, max: 7 days). Cosmetic results of the needle hole were very impressive both during the early postoperative period and during the 1-month follow-up (Photo 4).

\section{Discussion}

Single port cholecystectomy for gallbladder surgery offers less postoperative pain and better cosmetic results. However, there exist technical challenges with single port cholecystectomy. When operating on the abdomen, since there are 3 or 4 ports from a single device port, conflicts occur such as the overcrowding of tools. This situation lengthens the operation time and leads to technical problems in the operation. Various methods have been attempted to overcome this problem. One popular method is to make the percutaneous retraction of the fundus of the gallbladder by a placed suture. However, this process may be substantially limited for sac fundus retraction stabilization, and this method also has unwanted consequences, such as gallbladder injury during suturing. Van der Linden et al. [5] reported transcutaneous suture usage for the retraction of the gallbladder in SILC operations; they also reported pneumothorax for $2(2 / 36)$ patients as a complication. After the pneumothorax complications, they stopped using the suture and began to
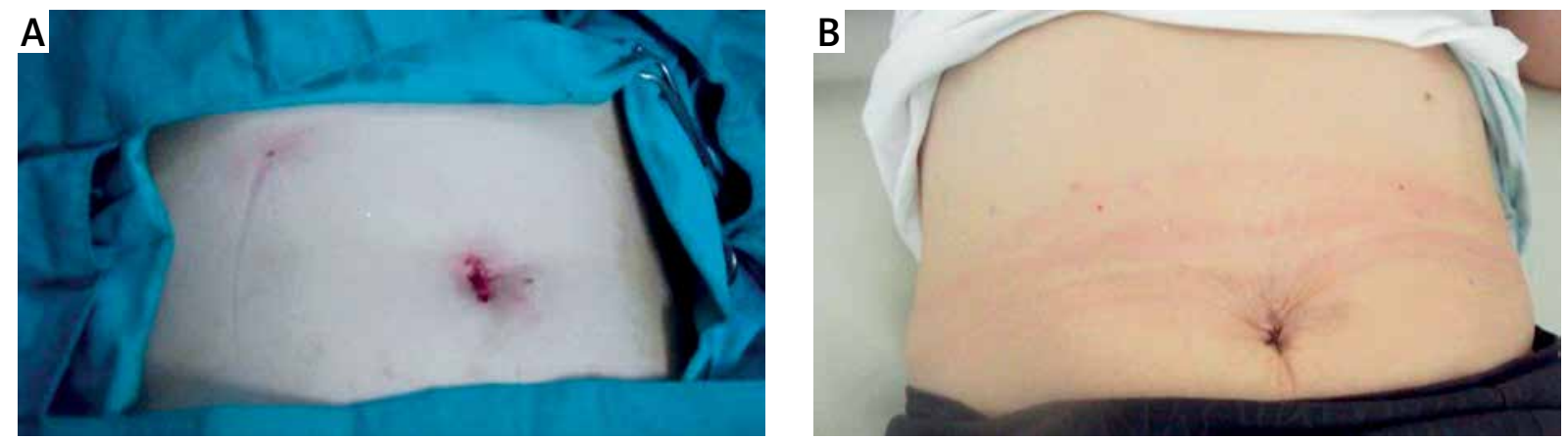

Photo 4. A - Image of the needle grasper entrance point and surgical field just after the operation. B - Image of the surgical field at post-operative day 20 
hang and retract the gallbladder with a clamp. Sinha and Yadav [6] reported a number of case series of Veress needle usage for the retraction of the gallbladder in transumbilical SILC operations. In this series, they inserted the Veress needle from the upper right quadrant and held the gallbladder with the help of this needle; they did not encounter any complications using this method. Yasumoto et al. [7] applied small forceps through a $3 \mathrm{~mm}$ trocar in the right subcostal area during SILS cholecystectomy, but the authors stated that there was a high risk of scarring.

The main difficulty of the SILS technique is to manipulate hand tools. The triangulation principle of the laparoscopic hand tool is not possible in SILS. Thus, SILS is not an ergonomic technique [8]. The use of a needle grasper in SILS cholecystectomy provided satisfactory results for both patients and surgeons. Inserting a needle into the abdominal cavity in the right subcostal area resulted in a small wound that vanished within 2 weeks, and most of the patients did not even notice it.

This novel technique was first reported by Komine et al. [9] and provided excellent cosmesis, as the surgeon used his left hand to configure a triangle of instruments. They also used this technique in cholecystectomies for benign gall-bladder diseases, similar to this study.

Some might think that this technique is not a kind of SILS, and an additional trocar might be necessary. But, who does not want the help of one more hand tool without making an incision? Actually, recently, similar retraction tools have been reported to facilitate operations. Some authors used a needle grasper with a $3 \mathrm{~mm}$ trocar, and some authors used EndoClose and a 2-mm atraumatic grasper without the use of a trocar $[7,10,11]$.

In our study, our new technique provides the surgeon with the following advantages:

1) Excellent cosmetic result without any skin incision due to using a needle grasper instead of a second trocar.

2) With the help of an assistant surgeon, the gallbladder was retracted from the fundus with the needle grasper, and it was able to be manipulated. It also easily revealed the Calot triangle.

3) Performing manipulative maneuvers by positioning the needle grasper facilitates the surgeon's dissection of the gallbladder.

Hanging and retracting the gallbladder in order to reveal the Calot triangle can be achieved with an additional tool, such as a percutaneous organ holder device. Some surgeons use a mini-grasper without a trocar to retract the gallbladder. With the help of needlescopic devices, operation time and scarring can be minimized [11]. These helper devices become significant and important for widening the indication of SILS operations [12].

In our study, the needle scar in the right subcostal region was negligible at the $3^{\text {rd }}$ day and completely disappeared by day 20 . Scar tissue related to the grasper needle was not observed in any patient. The needle grasper minimized tool crowding within the abdomen and facilitated the surgeon's work. Having the comfort of conventional 4-port laparoscopic procedures, we observed that following a short learning curve, the needle grasper could be manipulated easily, as in conventional laparoscopy, in our study. Entering the needle grasper in the right subcostal area, triangulation can be formed, and the gallbladder can be hung and manipulated easily. At the beginning of the study, though we were afraid of gallbladder perforation during hanging because of the sharp tip of the needle grasper, no perforation was observed in any of the patients. Instead, it was observed to hold the gallbladder even in the most difficult cases.

SILC operation time was reported as higher in almost all of the studies compared to conventional LC [13-16]. This difference may be related to the learning curve period. After the experience period, this operation time difference can be decreased, and similar results can be achieved [11, 17]. In our study, the mean operation time was $92.38 \mathrm{~min}$ in the first 29 patients and 81.56 min for the second 29 patients. After the experience period, our mean duration of operations decreased but was still longer than conventional LC.

In our study, we did not encounter any perioperative complications. In different studies, frequency of gallbladder perforation or bile leakage was reported as $2.2 \%$ and hemorrhage as $0.3 \%$ [18]. In another study, bile leakage frequency was reported as $0.66 \%$ [19]. In our study, we observed bile leakage in $1(1.69 \%)$ patient. This patient was admitted to our clinic with minor abdominal discomfort on the $6^{\text {th }}$ postoperative day, and $6 \times 5 \times 5 \mathrm{~cm}$ sized intra-abdominal free fluid at the pouch of Douglas was diagnosed with USG. This fluid was drained by USG-guided catheterization. Also, we did not encounter any port site hernia after a follow-up at 1 year. 
It was difficult to use this needle grasper in patients with high $\mathrm{BMI}$ and patients with acute cholecystitis; we think that its use should be restricted especially in acute cholecystitis. In patients with a high BMI (i.e., 38 and 43), the SILS technique could not be used because of the insufficient length of the tools.

\section{Conclusions}

In our opinion, the needle grasper will not only be helpful in cholecystectomy, but it can also be helpful in different kinds of single-incision laparoscopic operations. Since it is easy to use and does not require an incision or suture, it is a useful hand tool that should be included in the SILS laparoscopic set.

We observed in our study that SILS was a safe and effective technique whose most important advantages were the cosmetic result and high satisfaction.

Further randomized controlled trials are needed to evaluate the potential benefits of this new technique.

\section{Conflict of interest}

The authors declare no conflict of interest.

\section{References}

1. Cuschieri A. Minimal access surgery: the birth of a new era. J R Coll Surg Edinb 1990; 35: 345-7.

2. Rivas H, Varela E, Scott D. Single-incision laparoscopic cholecystectomy: initial evaluation of a large series of patients. Surg Endosc 2010; 24: 1403-12.

3. Liu JH, Etzioni DA, O'Connell JB, et al. The increasing workload of general surgery. Arch Surg 2004; 139: 423-8.

4. Navarra G, Pozza E, Occhionorelli S, et al. One-wound laparoscopic cholecystectomy. Br J Surg 1997; 84: 695.

5. van der Linden YT, Bosscha K, Prins HA, Lips DJ. Single-port laparoscopic cholecystectomy vs. standard laparoscopic cholecystectomy: a non-randomized, age-matched single center trial. World J Gastrointest Surg 2015; 7: 145-51.

6. Sinha R, Yadav AS. Transumbilical single incision laparoscopic cholecystectomy with conventional instruments: a continuing study. J Minim Access Surg 2014; 10: 175-9.

7. Yasumoto A, Tokumura H, Musya H, et al. Single-incision laparoscopic cholecystectomy with a small caliber forceps via additional $3 \mathrm{~mm}$ trocar in the right subcostal region. J Jpn Soc Endosc Surg 2012; 17: 535-8.

8. Chow A, Purkayastha S, Aziz O, Paraskeva P. Single-incision laparoscopic surgery for cholecystectomy: an evolving technique. Surg Endosc 2010; 24: 709-14.

9. Komine O, Suzuki H, Watanabe M, et al. Single-incision laparoscopic cholecystectomy with an additional needle grasper: a novel technique. J Nippon Med Sch 2015; 82: 43-9.
10. Altun H, Mantoglu B, Okuducu M, et al. Single-incision laparoscopic cholecystectomy: a new retraction technique. Acta Chir Belg 2013; 113: 311-3.

11. Gulpinar K, Ozdemir S, Ozis SE, et al. Single incision laparoscopic cholecystectomy by using a $2 \mathrm{~mm}$ atraumatic grasper without trocar. HPB Surg 2011: 761315.

12. Tagaya N, Kubota K. Reevaluation of needlescopic surgery. Surg Endosc 2012; 26: 137-43.

13. Marks J, Tacchino R, Roberts K, et al. Prospective randomized controlled trial of traditional laparoscopic cholecystectomy versus single-incision laparoscopic cholecystectomy: report of preliminary data. Am J Surg 2011; 201: 369-73.

14. Ma J, Cassera MA, Spaun GO, et al. Randomized controlled trial comparing single-port laparoscopic cholecystectomy and fourport laparoscopic cholecystectomy. Ann Surg 2011; 254: 22-7.

15. Lirici MM, Califano AD, Angelini P, Corcione F. Laparo-endoscopic single site cholecystectomy versus standard laparoscopic cholecystectomy: results of a pilot randomized trial. Am J Surg 2011; 202: 45-52.

16. Gangl O, Hofer W, Tomaselli F, et al. Single incision laparoscopic cholecystectomy (SILC) versus laparoscopic cholecystectomy (LC) - a matched pair analysis. Langenbecks Arch Surg 2011; 396: 819-24.

17. Bucher P, Pugin F, Morel P. From single-port access to laparoendoscopic single-site cholecystectomy. Surg Endosc 2010; 24: 234-5.

18. Antoniou SA, Pointner R, Granderath FA. Single incision laparoscopic cholecystectomy: a systematic review. Surg Endosc 2011; 25: 367-77.

19. Sinha R, Chandra S. Cystic duct leaks after laparoendoscopic single-site cholecystectomy. I Laparoendosc Adv Surg Tech A 2012; 22: 533-7.

Received: 5.02.2016, accepted: 23.03.2016 